\title{
Vibrational spectroscopy with neutrons: recent developments
}

\author{
Stewart F. Parker, Anibal J. Ramirez-Cuesta, Luke Daemen
}

\section{Published version information}

Citation: SF Parker, AJ Ramirez-Cuesta and L Daemen. "Vibrational spectroscopy with neutrons: recent developments." Spectrochimica Acta Part A, vol. 190 (2017): 518-523.

DOI: $10.1016 /$ j.saa.2017.09.057

(C)2017. This manuscript version is made available under the CC-BY-NC-ND 4.0 Licence.

This version is made available in accordance with publisher policies. Please cite only the published version using the reference above. This is the citation assigned by the publisher at the time of issuing the AAM. Please check the publisher's website for any updates. 


\title{
Vibrational spectroscopy with neutrons: recent developments.
}

\author{
Stewart F. Parker ${ }^{\mathrm{a} *}$, Anibal J. Ramirez-Cuesta ${ }^{\mathrm{b}}$, Luke Daemen ${ }^{\mathrm{b}}$
}

a ISIS Facility, STFC Rutherford Appleton Laboratory, Chilton, Didcot, OX11 OQX, UK

${ }^{\mathrm{b}}$ Chemical \& Engineering Materials Division, Oak Ridge National Laboratory, Oak Ridge, Tennessee 37831, USA

* Corresponding author.

E-mail address: stewart.parker@stfc.ac.uk (S.F. Parker).

This work was presented as a plenary lecture at ICAVS9, Victoria, Canada, $11^{\text {th }}-16^{\text {th }}$ June 2017.

\section{ABSTRACT}

In this short review, we will briefly summarise the differences between INS spectroscopy and conventional infrared and Raman spectroscopies. We will illustrate these with the current state-of-the art, using $\mathrm{C}_{70}$ as an example. The main focus of the article will be on the key advances in INS spectroscopy over the last ten years or so, that are driving new areas of research. The developments fall into three broad categories: (i) new sources, (ii) new and/or upgraded instrumentation and (iii) novel uses for existing instruments. For (i) we summarise the new neutron sources that are now, or will be, operating. For (ii) we show the capabilities of new or upgraded instruments. These offer unprecedented levels of sensitivity: submillimole quantities of hydrogen can be measured and millimole quantities of low cross section materials. Recent work on hexahalo metallates and adsorbed $\mathrm{CO}_{2}$ is used to demonstrate what is now feasible. For (iii), instruments that were designed for studies of magnetism, are now being used for molecular spectroscopy, especially for catalysts. This is illustrated with work on $\mathrm{CuH}$ and methanol synthesis catalysts.

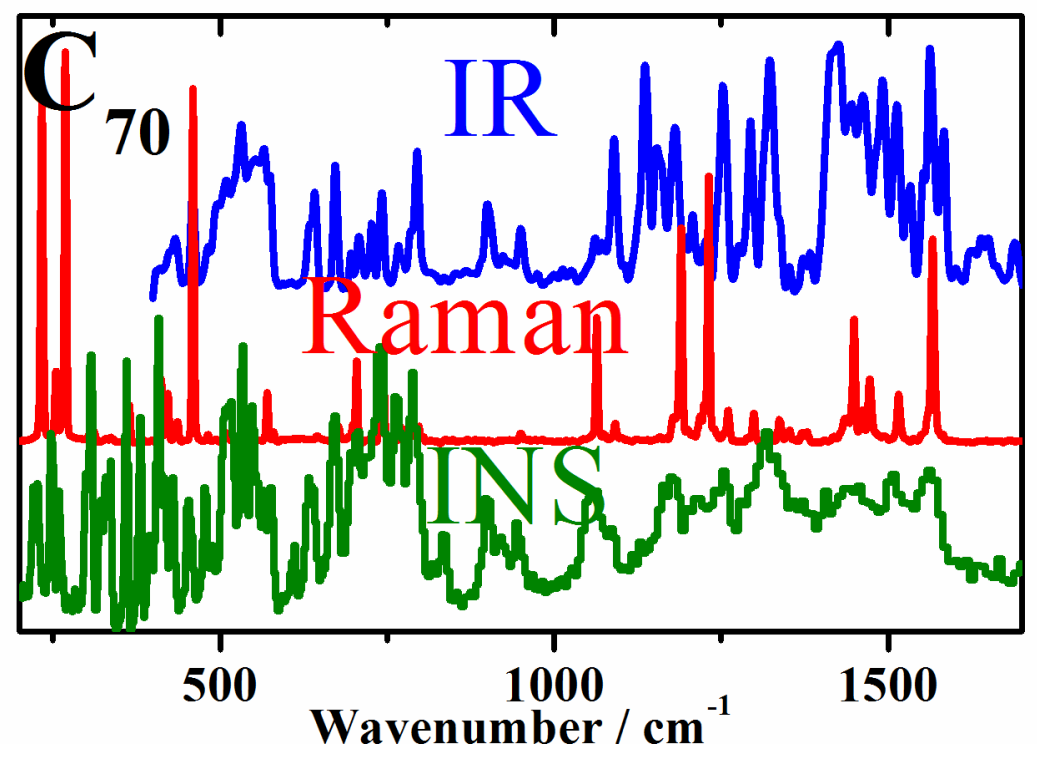




\section{The current state-of-the art}

In the International Conference on Advanced Vibrational Spectroscopy (ICAVS) series, vibrational spectroscopy with neutrons (inelastic neutron scattering spectroscopy, INS, [1]) was last reviewed [2] at ICAVS5 (Melbourne, Australia, $12^{\text {th }}-17^{\text {th }}$ July 2009). In this short review, we will briefly summarise the differences between INS spectroscopy and conventional infrared and Raman spectroscopies. We will illustrate these with the current state-of-the art. The main focus of the article will be on developments in instrumentation since the last review [2] that are opening new areas of investigation for INS spectroscopy.

The use of neutrons to carry out vibrational spectroscopy has several advantages. Amongst these are that there are no symmetry-based selection rules in INS spectroscopy so, in principle, all modes are observable. This arises because neutrons are scattered by atomic nuclei via the strong force, in contrast to infrared and Raman spectroscopies where the interaction is between photons and electrons. Some of the other consequences of the use of neutrons are seen from a simplified form of the expression for the scattered intensity [1]:

$$
S\left(Q, n \omega_{i}\right) \propto \frac{\left(Q U_{i}\right)^{2 n}}{n !} \exp \left(-\left(Q U_{\mathrm{Tot}}\right)^{2}\right) \sigma_{\omega_{i}}
$$

Where $\omega_{i}$ is the $i$ th mode at transition energy $\omega, n=1$ for a fundamental, 2 for a first overtone or binary combination, 3 for a second overtone or ternary combination etc..., $Q$ is the momentum transfer, $U_{i}$ is the root mean square displacement of the atoms in the mode and $\sigma$ is the inelastic scattering cross section of the atom. $U_{T o t}$ is the total root mean square displacement of all the atoms in all the modes, (both internal and external). (A more detailed theoretical description can be found in [1]).

The left-hand side of equation (1) is the measured intensity, $S(Q, \omega)$. This depends on two variables: the transition energy and the momentum transfer $\left(Q,|Q|=Q, \AA^{-1}\right)$ :

$$
\boldsymbol{Q}=\boldsymbol{k}_{\boldsymbol{i}}-\boldsymbol{k}_{f} \text { where } k \equiv 2 \pi / \lambda
$$

$k\left(\AA^{-1}\right)$ is the wavevector; $\lambda(\AA)$ is the wavelength of the neutron, $i$ and $f$ refer to the incident and final neutron respectively. The momentum dependence arises because a neutron has mass (1.009 amu) and any scattering event will involve a change in momentum (since the neutron will change direction) and may also involve a change in energy. The dependence of the intensity on $Q$ is the major difference between INS and infrared and Raman spectroscopies, where the modes are observed at (almost) $Q=0$.

The exponential term is a Debye-Waller factor and its magnitude is, in part, determined by the thermal motion of the system. This can be reduced by cooling the sample and so spectra are typically recorded below $30 \mathrm{~K}$.

The last term is the incoherent scattering cross section of the elements in mode $\omega$, which is both element and isotope dependent. For normal hydrogen, ${ }^{1} \mathrm{H}$, it is 80.2 barns $(1 \mathrm{barn}=1 \times$ $10^{-28} \mathrm{~m}^{-2}$ ) whereas for deuterium, ${ }^{2} \mathrm{H}$, and for virtually all other elements it is $<5$ barns. Note 
that for ${ }^{1} \mathrm{H}$, the combination of the large cross section and that it has the largest amplitude of vibration (since it is the lightest atom), means that there is a strong "propensity" rule and that in a hydrogenous material, the observed modes are those that involve motion of hydrogen.

While materials containing ${ }^{1} \mathrm{H}$ are particularly advantageous for INS spectroscopy, they are not essential. Fig. 1 compares the infrared, Raman and INS spectra of $\mathrm{C}_{70}$ [3] in the internal mode region. The complementarity between the techniques is clear; modes that are weak or absent in one, appear clearly in another. Note that the INS spectrum includes all the modes, as there are no symmetry-based selection rules.

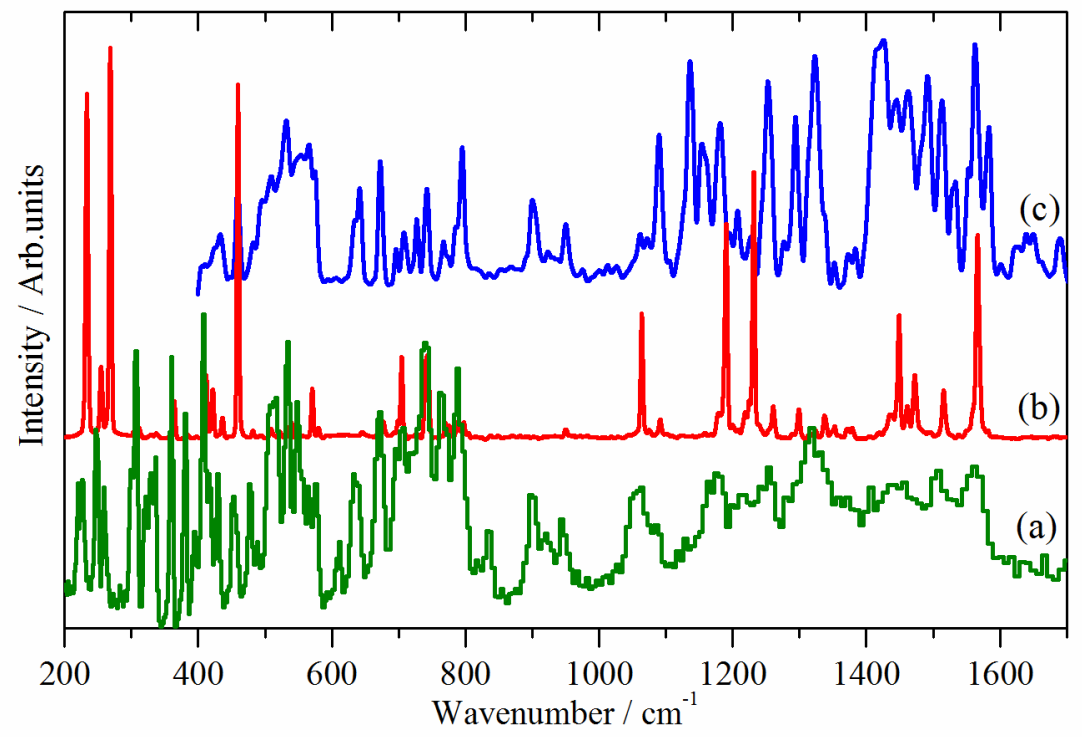

Fig. 1. Vibrational spectra of $\mathrm{C}_{70}$ [3]: (a) INS spectrum at $7 \mathrm{~K}$, (b) Raman spectrum at $7 \mathrm{~K}$ using 785 excitation, after correction for the instrument response and a sloping baseline, recorded simultaneously with the INS spectrum. (c) Infrared spectrum at $113 \mathrm{~K}$ using ATR after baseline correction.

It is worth noting that equation (1) is purely dynamic, thus any method that can provide the transition energies and the atomic displacements of a vibrational mode can be used to generate the INS spectrum. Comparison of observed and calculated INS spectra provides a very stringent test of a model and was used to show that all the literature assignments of $\mathrm{C}_{70}$ were, at least partially, incorrect. Fig. 2 shows a comparison of the observed spectrum of $\mathrm{C}_{70}$ and that calculated by periodic density functional theory (DFT). It can be seen that the agreement is almost exact for both the transition energies and the intensities. This level of concordance requires considerable effort, but demonstrates what is achievable. The complementarity between experiment and theory is one of the great strengths of neutron scattering in general and of INS spectroscopy in particular. 


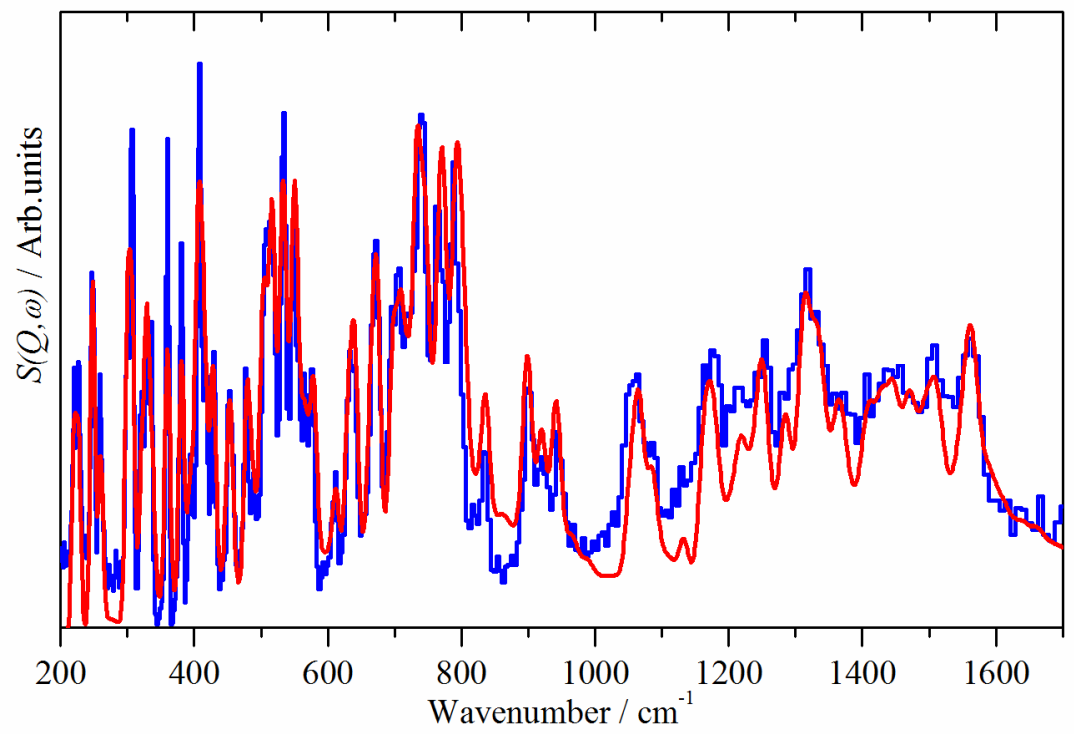

Fig. 2. Comparison of the TOSCA INS spectrum of $\mathrm{C}_{70}$ at $7 \mathrm{~K}$ (blue) with that calculated for the solid state by CASTEP (site symmetry $C_{\mathrm{s}}$ ) after individual scaling of the modes and inclusion of the phonon wings. Reproduced from [3] with permission of Wiley-VCH.

\section{Recent developments}

The key advances in INS spectroscopy over the last ten years or so, fall into three broad categories: (i) new sources, (ii) new and/or upgraded instrumentation and (iii) novel uses for existing instruments. Each of these will be considered in more detail in the following sections.

\subsection{New sources}

Neutron scattering can only be carried out at dedicated facilities [4]. There are $~ 40$ of these around the world, with the majority in Europe and North America. Most neutron sources are reactors. Research reactors are designed to deliver thermal neutrons with a peak flux at $\sim 200 \mathrm{~cm}^{-1}$ and they usually have little intensity at $>800 \mathrm{~cm}^{-1}$. This is typified by the newly-installed beryllium-filter option [5] on TAIPAN at ANSTO (Lucas Heights, Australia). The exception to this rule is the Institut Laue-Langevin (ILL) [6] at Grenoble in France, which uses a block of graphite heated to $\sim 2000 \mathrm{~K}$ to generate hot neutrons from thermal neutrons. For vibrational spectroscopy a wide energy range is required and spallation sources are pre-eminent in this area. These have the added advantages of intrinsically low backgrounds and do not use fissile material. From its first operation in 1985, the ISIS Facility [7] at Chilton in the UK has been the world-leader. In the last decade or so, the SNS (Oak Ridge, USA) [8] and J-PARC (Tokai, Japan) [9] have started user operations. These nextgeneration sources offer unprecedented levels of flux and benefit from the advances in neutron optics that have occurred over the last 20 years. The institutions have three (SNS) and two (J-PARC) INS spectrometers.

An on-going development is the construction of the European Spallation Source (ESS) [10] at Lund in southern Sweden. This is scheduled to be fully operational in 2025 and will 
have three INS spectrometers. One of these, VESPA [11], will outperform all existing instruments of its type. This because the flux-on-sample will be a factor of 10 larger than for any other instrument, with a resolution that is at least as good as the best currently available.

\subsection{New and/or upgraded instrumentation}

The arrival of new sources has resulted in new instruments and spurred the upgrading of existing ones. For vibrational spectroscopy, the key instruments are IN1-Lagrange (ILL, France) [12], TOSCA, (ISIS, UK) [13] and VISION (SNS, USA) [14].

Fig. 3 shows what is now achievable on IN1-Lagrange. This shows the INS spectrum of only $17 \mathrm{mg}$ of 2,5-diiodothiophene, a commonly used resolution and calibration standard [15]. This sample contains only $0.1 \mathrm{mmol}$ of hydrogen; for the previous generation of instruments, the rule-of-thumb was that $10 \mathrm{mmol}$ of hydrogen was the minimum required for an acceptable spectrum.

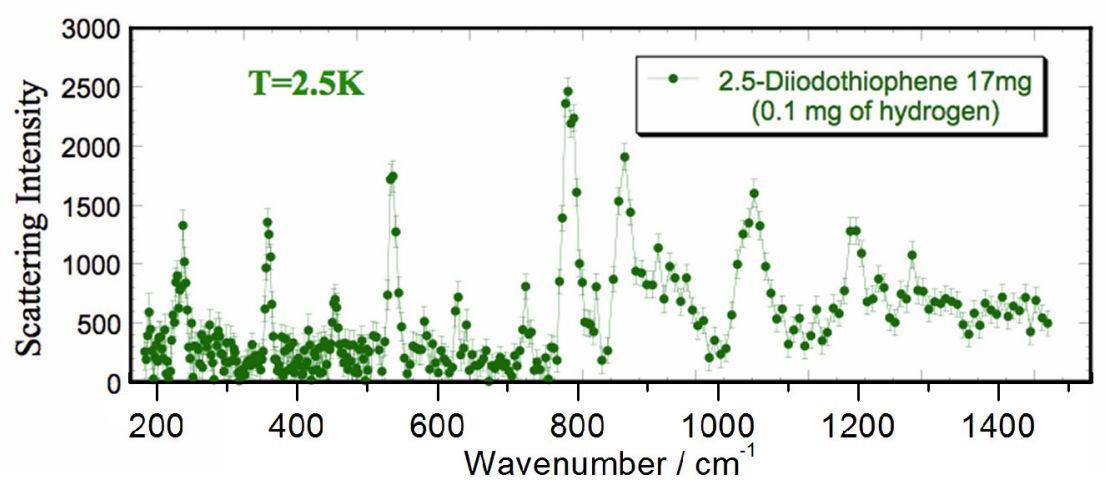

Fig. 3. The spectrum of a $17 \mathrm{mg}$ sample of 2,5-diiodothiophene $\left(\mathrm{C}_{4} \mathrm{H}_{2} \mathrm{I}_{2} \mathrm{~S}\right)$ containing 0.1 mg of hydrogen) obtained as a difference of the scattering intensities measured on IN1Lagrange with the sample and with an empty sample can. Both runs in the shown energy transfer range took less than eight hours each with the $\mathrm{Cu} 220$ 2D-focussing monochromator. Reproduced from [12] with permission of the Institute of Physics.

Fig. 4 shows a series of spectra for the complexes $\mathrm{Mg}_{2}\left[\mathrm{FeH}_{6}\right]$ and $\mathrm{K}_{2}[\mathrm{PtX} 6](\mathrm{X}=\mathrm{H}, \mathrm{Cl}$. $\mathrm{Br}$ I). At room temperature, the complex ions are octahedral and the structures are face centred cubic (or nearly so), which results in them having several modes that are inactive in both the Raman and infrared spectra $[16,17]$. The spectra show the expected trends: the transition energies decrease with increasing atomic number and all the modes are present. The figure illustrates how the technique has evolved with time. Fig. $4 \mathrm{a}$ and $4 \mathrm{~b}$ were recorded with a first generation spectrometer, TFXA [1] at ISIS, Fig. 4c with the second generation spectrometer TOSCA after its recent upgrade to increase the incident flux $\sim$ tenfold [13] and Fig. 4d with VISION, which is currently the world's best INS instrument of this type.

The advances that have been made are shown by the filled circles on the right of the figure. The area is proportional to the product of the total scattering cross section and the amplitude of vibration (equation (1)) for the highest energy mode, so represent the relative sensitivity for $\mathrm{H}, \mathrm{Cl}, \mathrm{Br}$ and $\mathrm{I}$. As noted earlier, $\mathrm{H}$ is the most favourable case, so even with 
an early spectrometer a high signal-to-noise ratio was obtained. $\mathrm{Cl}$ is a much less favourable case, and only the combination of the unusually large cross section, 16 barns, and the use of a large sample made the measurement feasible [17]. Even so, the signal-to-noise ratio is inferior to that of Fig. 4a. For $\mathrm{X}=\mathrm{Br}$, Fig 4c, the sensitivity is a factor of four smaller than for $\mathrm{X}=\mathrm{Cl}$ and the sample size was less than one-third that of the chloro complex.

Nonetheless, the signal-to-noise ratio is roughly comparable in Fig. 4b and 4c. In Fig. 4d using VISION, the scattering power is almost a factor of two smaller for $\mathrm{X}=\mathrm{I}$ as compared to $\mathrm{X}=\mathrm{Br}$, and for almost the same sample size (12 mmol $\mathrm{Br}$ vs $10 \mathrm{mmol} \mathrm{I})$, the signal-tonoise ratio is as good as that for the hydrogenous sample.

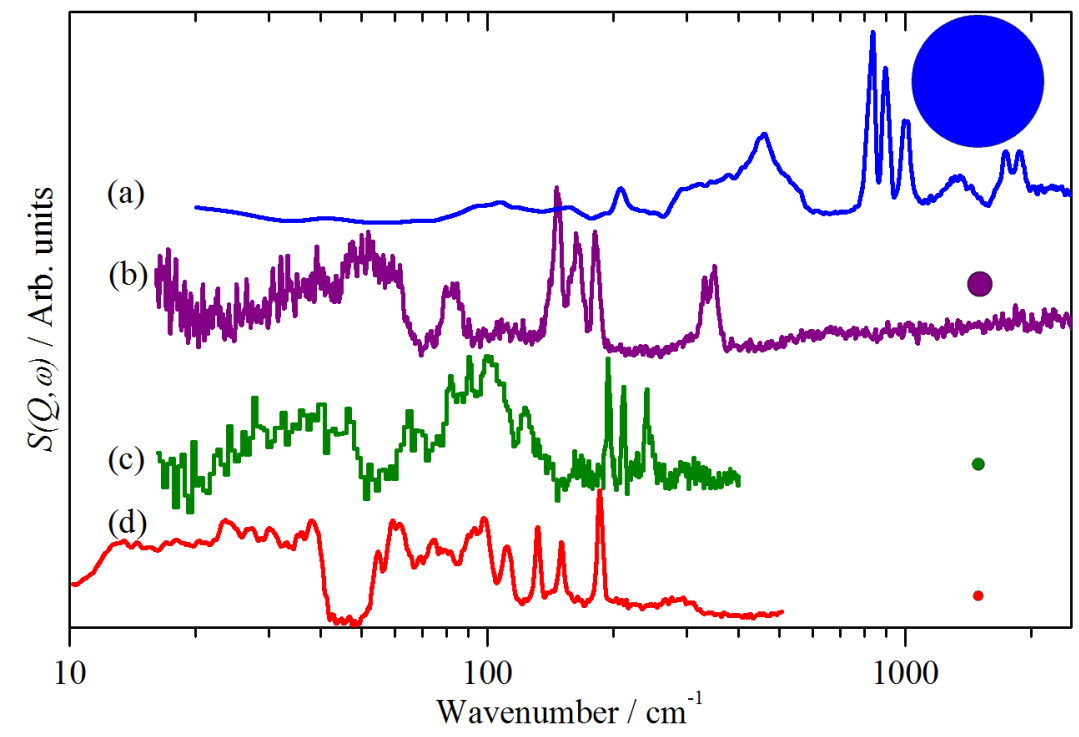

Fig. 4 INS spectra of the octahedral complexes $\mathrm{Mg}_{2}\left[\mathrm{FeH}_{6}\right]$ (a) and $\mathrm{K}_{2}\left[\mathrm{PtX}_{6}\right](\mathrm{X}=\mathrm{Cl}, \mathrm{Br}, \mathrm{I})$ (b), (c) and (d) respectively. The filled circles on the right are proportional to the product of the total scattering cross section and the amplitude of vibration for the highest energy mode, so represent the relative sensitivity for $\mathrm{H}, \mathrm{Cl}, \mathrm{Br}$ and $\mathrm{I}$. (a) and (b) were recorded on TFXA, (c) on the upgraded TOSCA and (d) on VISION.

The ability to measure spectra from weakly scattering systems, was used to investigate the interactions of $\mathrm{CO}_{2}$ with nanoporous $\mathrm{S}$ - and $\mathrm{N}$-doped polymer-derived carbon and commercial wood-based carbon [18]. Fig. 5 compares the INS spectra of ice and solid $\mathrm{CO}_{2}$ with that of $\mathrm{CO}_{2}$ adsorbed on oxidized carbon $(\mathrm{C}-\mathrm{AO})$ before and after reaction at room temperature. The characteristic bending mode of $\mathrm{CO}_{2}$ at $645 \mathrm{~cm}^{-1}$ is clearly visible, even with only $2.5 \mathrm{mmol}$ present. While the spectrum of $\mathrm{CO}_{2}$ when initially adsorbed resembles that of the bulk, after reaction the lattice modes have gone and it is most likely adsorbed on the surface of the sample. Water is also produced and the difference in the slope of the leading edge of the librational modes at $\sim 550 \mathrm{~cm}^{-1}$ is consistent with it being subjected to stronger restrictions imposed by their interactions in the pore system.

Catalysis is a major area of activity for INS spectroscopy $[19,20]$. To date, all the systems studied have involved hydrogenous adsorbates. This work shows that studies of nonhydrogenous adsorbates are now feasible. 


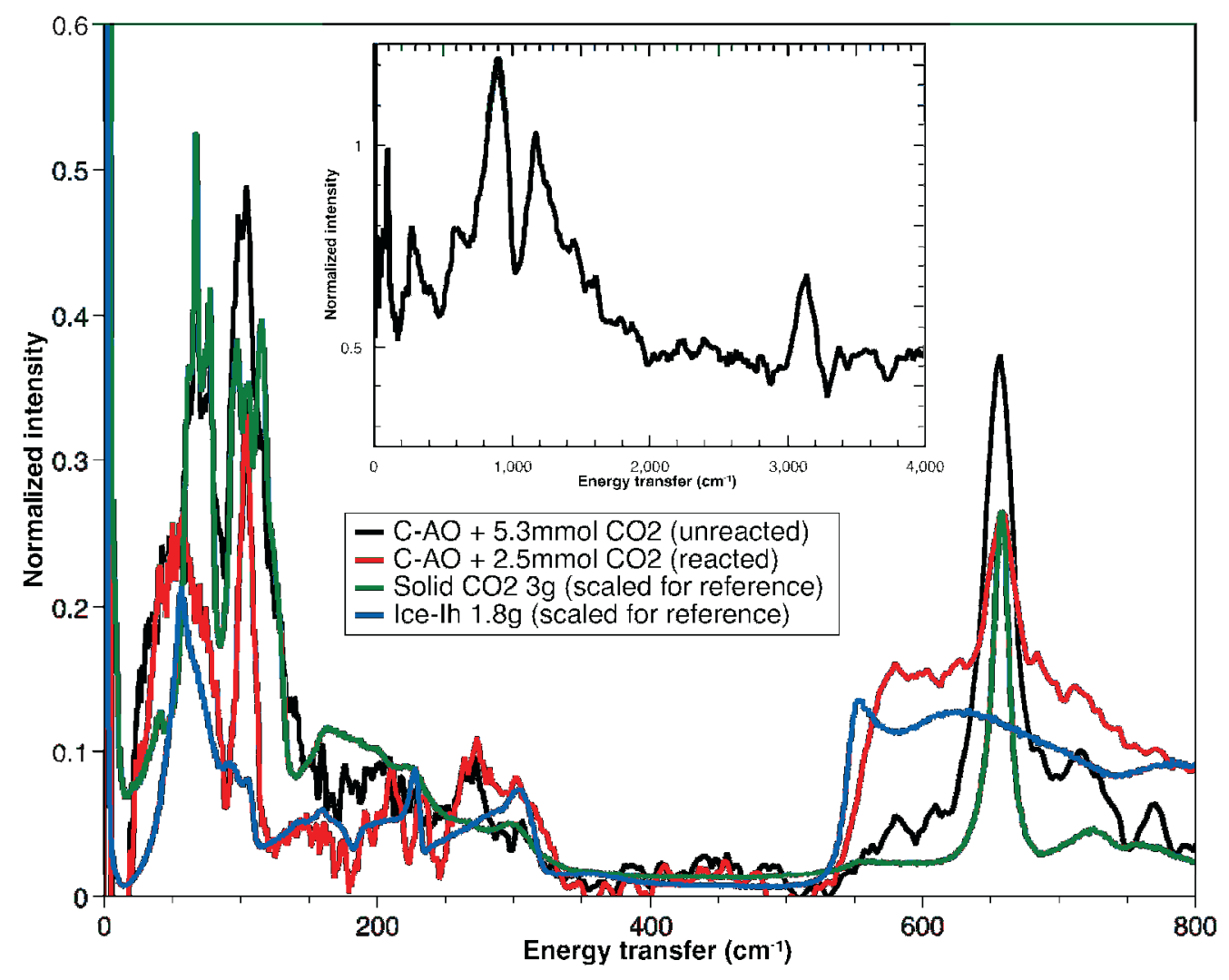

Fig. 5. The difference INS spectra before and after $\mathrm{CO}_{2}$ dosing in $\mathrm{C}-\mathrm{AO}$, in comparison with the reference spectra for bulk solid $\mathrm{CO}_{2}$ and $\mathrm{H}_{2} \mathrm{O}$. The signal from the background and the blank C-AO has been subtracted. Inset shows the INS spectrum of the blank C-AO before dosing (note that the energy scale is different). Reproduced from [18] with permission of Elsevier.

\subsection{Novel uses for existing instrumentation}

At neutron scattering facilities, typically $30-50 \%$ of all beam time is used for studies of magnetism. In the 'mid-infrared' region, the proportion is even higher. INS instruments used for magnetism are generally of the type known as 'direct geometry'. As explained in more detail elsewhere $[1,22]$, these instruments use a chopper, or a monochromator, to select a (nominally) single energy from an incident white beam. Either by moving a single detector through a range of angles or by having an array of detectors to cover a large angular range (modern instruments have around $\pi$ sterardians of coverage), they enable a wide range in $(Q, \omega)$ space to be measured. An example is shown in Fig. 6. The reason for the need for both $Q$ and $\omega$ information is that magnetic transitions are often highly dispersed (i.e. the transition energy varies considerably with wavevector) and have a very strong form factor (i.e. the intensity falls off rapidly with increasing $Q$ ), in contrast to that of phonons (Equation (1)) and this provides a means to distinguish the two behaviours.

By comparison, the instruments that generated the INS spectra of Figs. 1-5, IN1-Lagrange, TOSCA and VISION, are known as 'indirect geometry' instruments. These have a specific 
trajectory through $(Q, \omega)$ space, and all instruments of this type follow trajectories that lie on or between the solid lines on Fig. 6 .

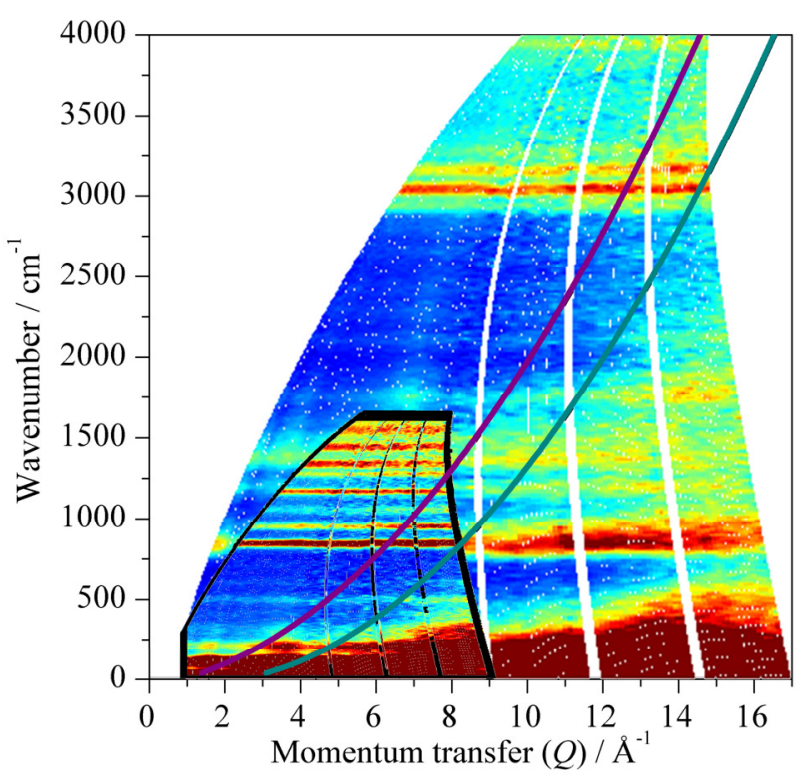

Fig. 6. $(Q, \omega)$ plot of iodomethane at $20 \mathrm{~K}$ recorded on MAPS at ISIS with incident energies of 4840 (large segment) and $2017 \mathrm{~cm}^{-1}$ (small segment). The solid lines are the trajectories of an indirect geometry instrument with a final energy of $\sim 40 \mathrm{~cm}^{-1}$ for scattering angles of $45^{\circ}$ (purple trace) and $135^{\circ}$ (olive trace). Reproduced from [16] with permission of the Institute of Physics.

The crucial difference between direct and indirect geometry spectrometers is that the former can always access smaller $Q$ than the latter [16]. This is shown in Fig. 7 which shows the $(Q, \omega)$ plot for iodomethane (methyl iodide, $\left.\mathrm{CH}_{3} \mathrm{I}\right)$ recorded with incident energies $(\mathrm{Ei})$ of 4840 and $2017 \mathrm{~cm}^{-1}$. The parabolic lines show the path through $(Q, \omega)$ space for an indirect geometry instrument with scattering angles of $45^{\circ}$ (left) and $135^{\circ}$ (right) and, critically, high energy $\left(>2000 \mathrm{~cm}^{-1}\right)$ transfer means high momentum transfer. From Equation (1), higher order transitions, i.e. $n \geq 2$, become significant at high $Q$, because they vary as $Q^{2 n}$, and phonon wings (combinations between internal and external modes) become intense. The problem is compounded by the reduction of intensity caused by the Debye-Waller factor, which is larger at high $Q$. These two effects mean that the fundamental transitions are mostly overwhelmed by the more intense higher order processes, making them undetectable. By only selecting the low $Q$ detectors of a direct geometry spectrometer, $\mathrm{C}-\mathrm{H}$ and $\mathrm{O}-\mathrm{H}$ fundamentals are easily seen. This is demonstrated for iodomethane in Fig. 7, where only the small $Q$ data of Fig. 6 is compared to the TOSCA spectrum, Fig. 7a. Based only on the TOSCA data, it would be natural to assign the arrowed peak at $3043 \mathrm{~cm}^{-1}$ to the unresolved $\mathrm{C}-\mathrm{H}$ stretch modes. Comparison with the MAPS spectrum, Fig. 7d, which has considerably better signalto-noise ratio and shows an additional peak at $2948 \mathrm{~cm}^{-1}$ (arrowed). It is actually this peak that is the unresolved C-H stretch modes; the peaks at $3043,3161,3272 \mathrm{~cm}^{-1}$ are 
combinations: $\mathrm{C}-\mathrm{H}$ stretch + libration, $\mathrm{C}-\mathrm{H}$ stretch $+2 \times$ libration, $\mathrm{C}-\mathrm{H}$ stretch $+3 \times$ libration respectively. (The librational mode is at $112 \mathrm{~cm}^{-1}$ ).

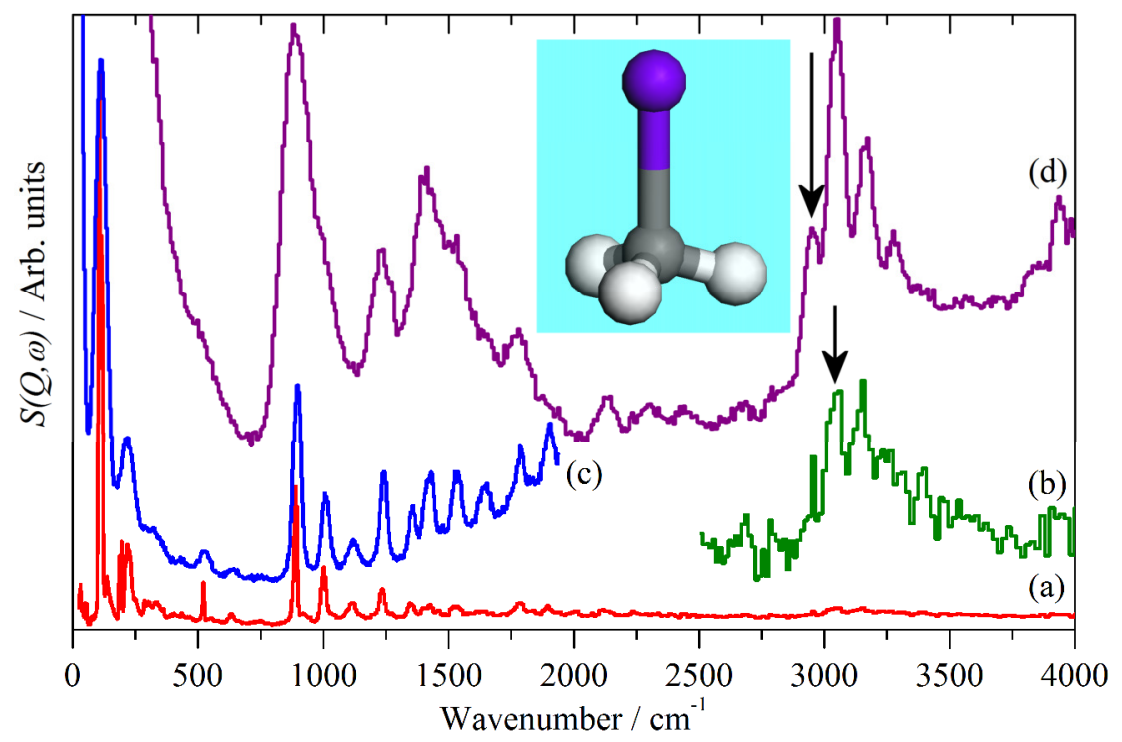

Fig. 7. INS spectra of iodomethane recorded at $20 \mathrm{~K}$ on (a) TOSCA, (b) TOSCA $\times 10$ ordinate expansion of the $2500-4000 \mathrm{~cm}^{-1}$ region, (c) and (d) MAPS with incident energies of 4840 and $2017 \mathrm{~cm}^{-1}$ respectively. (c) and (d) are the data shown in Fig. 6 summed for the detectors at $4 \leq Q \leq 10 \AA^{-1}$. Reproduced from [16] with permission of the Institute of Physics.

The ability to access low $Q$ has been extensively exploited for studies of catalysts [20], particularly those with hydrocarbonaceous overlayers, that may (e.g. coke) or may not be detrimental. The processes studied have included: methyl chloride synthesis [23], methane reforming [24,25], Fischer-Tropsch synthesis [26], methyl methacrylate synthesis [27] and methanol-to-hydrocarbons [28-30].

Methanol is one of the largest-scale commodity chemicals: in 201570 million tonnes were used. It has been made industrially for more than 50 years from synthesis gas $\left(\mathrm{H}_{2}, \mathrm{CO}, \mathrm{CO}_{2}\right)$ using a $\mathrm{Cu} / \mathrm{ZnO} / \mathrm{Al}_{2} \mathrm{O}_{3}$ catalyst at $250{ }^{\circ} \mathrm{C}$ and 50-100 bar. Laboratory studies [31] have shown that there is a substantial amount of hydrogen locked-in sub-surface in the copper component. One possibility is that this is present as $\mathrm{CuH}$, copper (I) hydride. $\mathrm{CuH}$ has been known for almost 200 years [32], but has only recently been fully characterized [33-36]. One of the major puzzles was why $\mathrm{CuH}$ synthesized by aqueous methods (denoted as $\mathrm{CuH} / \mathrm{Würtz}$ ) differed so much from $\mathrm{CuH}$ synthesized from organic donor solvents (denoted as $\mathrm{CuH} /$ pyridine). Total scattering neutron diffraction [34] showed that $\mathrm{CuH}$ was the same in the products from both routes. However, INS spectroscopy in the $\mathrm{C}-\mathrm{H} / \mathrm{O}-\mathrm{H}$ stretch region using a direct geometry instrument (MAPS) showed distinct differences between the products.

The INS spectrum of dried $\mathrm{CuH} / \mathrm{Würtz}$ recorded on a direct geometry spectrometer is shown in Fig. 8 and three peaks are apparent at 2054, 2912, and $3249 \mathrm{~cm}^{-1}$. From Equation (1) it can be shown [37] that $S(Q, n \omega)$ goes through a maximum, $Q_{\max }$, that differs for each value of $n$ (it increases with increasing $n$ ). From Fig. 9, it is obvious that $Q_{\max } 1060<Q_{\max }$ $2054<Q_{\max } 2912 \mathrm{~cm}^{-1}$ and the assignment that they correspond to the $n=1,2,3$ transitions 
of $\mathrm{CuH}$ naturally follows. From the first two transitions, the harmonic frequency is calculated as $\omega_{e}=1122 \mathrm{~cm}^{-1}$ and the anharmonicity $2 \omega_{e} x_{e}=66 \mathrm{~cm}^{-1}$. Using these values, the $n=3$ transition is predicted at $2970 \mathrm{~cm}^{-1}$ and the $2912 \mathrm{~cm}^{-1}$ peak is assigned accordingly. This leaves the $3249 \mathrm{~cm}^{-1}$ peak unassigned. The transition energy is consistent with that of the $\mathrm{O}-\mathrm{H}$ stretch of a surface $\mathrm{Cu}-\mathrm{OH}$ group and is so assigned. The assignment to residual water is untenable as the $\mathrm{H}-\mathrm{O}-\mathrm{H}$ bend would be expected at $\sim 1600 \mathrm{~cm}^{-1}$ and there are no features in this region.

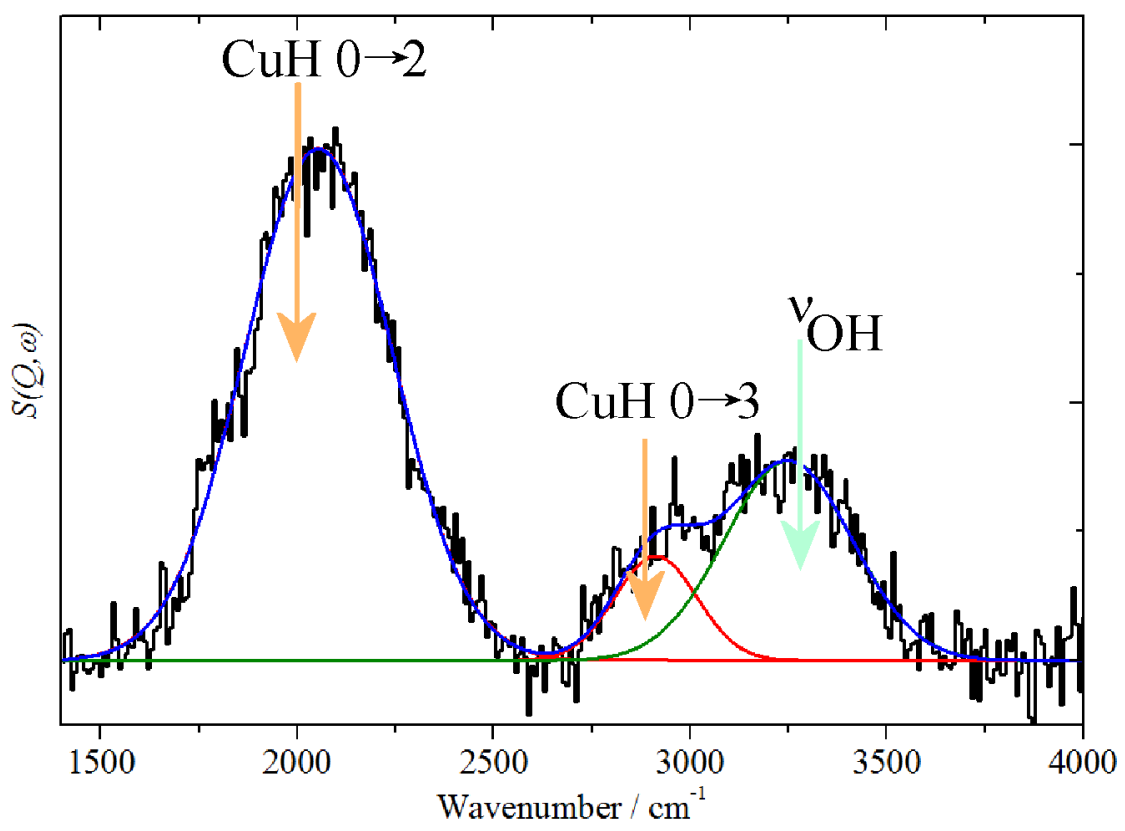

Fig. 8. Curve-resolved (after baseline correction) INS spectrum of the dried $\mathrm{CuH} / \mathrm{Würtz}$ product in the high-energy region recorded on MAPS (with an incident energy of $4840 \mathrm{~cm}^{-1}$ ) at $20 \mathrm{~K}$. Note the absence of any peak at $\sim 1600 \mathrm{~cm}^{-1}$. Reproduced from [34] with permission of the American Chemical Society. 


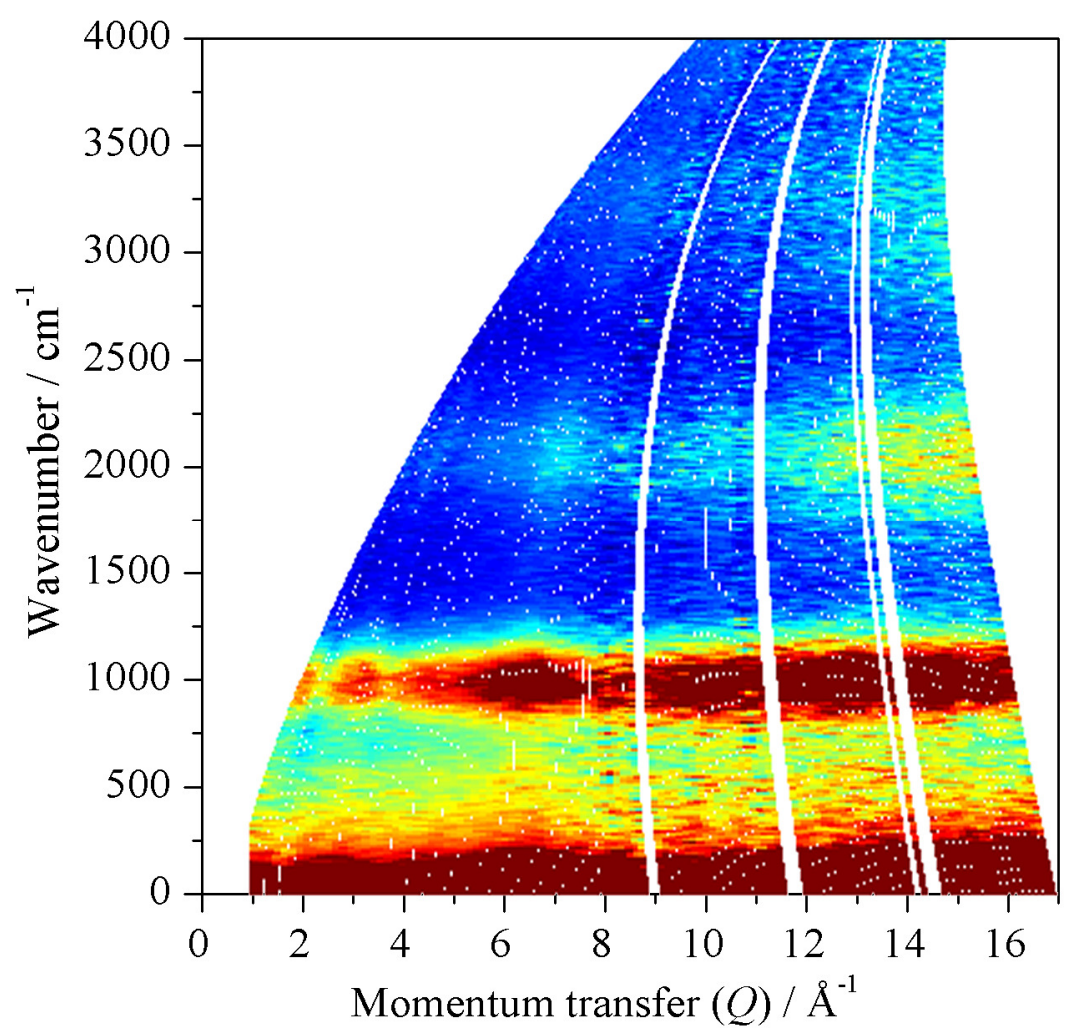

Fig. 9. $S(Q, \omega)$ map of the dried $\mathrm{CuH} /$ Würtz product recorded on MAPS at $20 \mathrm{~K}$ with an incident energy of $4840 \mathrm{~cm}^{-1}$. Reproduced from [34] with permission of the American Chemical Society.

Measurement of the INS spectrum of $\mathrm{CuH} /$ pyridine in the same region, showed no evidence for hydroxyls, only the unresolved $\mathrm{C}-\mathrm{H}$ stretch modes of coordinated pyridine. The spectra show that $\mathrm{CuH}$, synthesized by aqueous routes, consists of relatively large (several hundred nanometers) nanoparticles of a poorly crystalline core of nonstoichiometric $\mathrm{CuH}$ capped with a monolayer of hydroxyls and surrounded by water molecules. For $\mathrm{CuH} /$ pyridine, a model of a very small, poorly crystalline core of nonstoichiometric $\mathrm{CuH}$ capped with a monolayer of pyridine is indicated. Computational studies show that both structures are stable. The difference in the surface chemistry of $\mathrm{CuH}$ formed by aqueous and non-aqueous routes provides a rationale for their differing behaviors. Functionalization and optimization of nanoparticles by manipulation of the surface layer is a topic of considerable interest, as it potentially provides a means to tailor the properties of the system. $\mathrm{CuH}$ provides a particularly clear example of how the nature of an adsorbed layer on a nanoparticle surface determines its properties.

Fig. 10 shows difference (reacted - clean) INS spectra of a $\mathrm{Cu} / \mathrm{ZnO}$ catalyst after methanol synthesis at 6.31 bar and $523 \mathrm{~K}$ in $\mathrm{CO} / \mathrm{H}_{2}$, Fig $10 \mathrm{a}$, and $\mathrm{CO}_{2} / \mathrm{H}_{2}$, Fig 10b, [38]. The peaks labelled $\mathrm{M}$ in both spectra at 2940,1450, 1160 and $95 \mathrm{~cm}^{-1}$ are assigned as the $\mathrm{C}-\mathrm{H}$ stretch, the $\mathrm{C}-\mathrm{H}$ deformations, methyl rock and methyl torsion respectively of chemisorbed methoxy. For the reaction in $\mathrm{CO}_{2} / \mathrm{H}_{2}$, formate is also present on the surface: the in-plane $\mathrm{C}-\mathrm{H}$ deformation mode is clearly seen at $1375 \mathrm{~cm}^{-1}$, the weaker out-of-plane deformation is just visible at $1055 \mathrm{~cm}^{-1}$. Unfortunately, the mode of formate coordination (mono- or bidentate) 
cannot be distinguished by INS spectroscopy as the characteristic modes are the symmetric and asymmetric $\mathrm{C}-\mathrm{O}$ stretches which are invisible to INS. For the specific example of adsorbed formate, comparison of observed and calculated INS spectra is also not helpful because the $\mathrm{C}-\mathrm{H}$ stretch and deformation modes (which are the modes observed by INS) occur at the same transition energy, irrespective of the mode of binding (mono- or bidentate). A parallel computational study [39] showed that the bidentate form bridging two copper atoms is the lowest energy structure on all the low index copper surfaces. Another difference between the reactions is the presence of hydroxyls, as shown by the bands at 3430 and 915 $\mathrm{cm}^{-1}$ for the $\mathrm{O}-\mathrm{H}$ stretch and bend respectively. The transition energy of the hydroxyl bending mode suggests that the hydroxyls are on the $\mathrm{Cu}$ component rather than the $\mathrm{ZnO}$. Thus there are distinct differences between the behaviour of the catalyst depending on the feedstock.

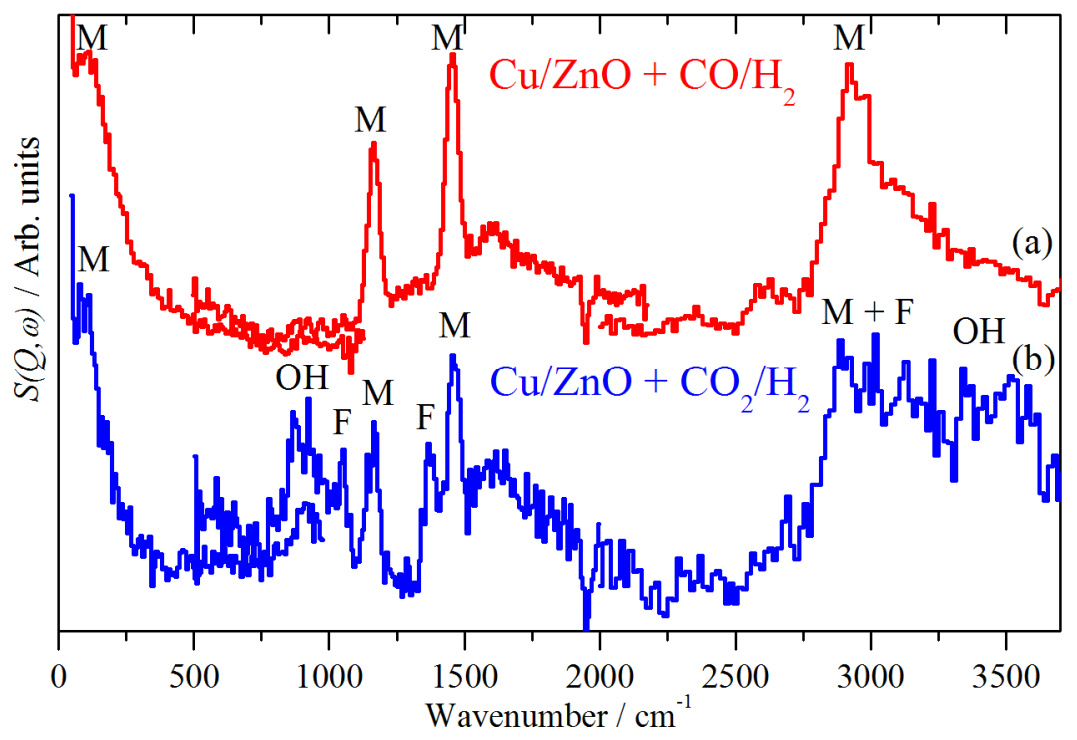

Fig. 10 Difference INS spectra recorded on MAPS at ISIS of $\mathrm{Cu} / \mathrm{ZnO}$ after methanol formation with a) $\mathrm{CO} / \mathrm{H}_{2}$ and b) $\mathrm{CO}_{2} / \mathrm{H}_{2}$ feed. Peaks labelled $\mathrm{M}$ are methoxy, $\mathrm{F}$ are formate and $\mathrm{OH}$ are hydroxyls on $\mathrm{Cu}$. The region $4000-2000 \mathrm{~cm}^{-1}$ was recorded with an incident energy $\left(E_{i}\right)=4840 \mathrm{~cm}^{-1}, 2000-500 \mathrm{~cm}^{-1}$ with $E_{i}=2420 \mathrm{~cm}^{-1}$ and $500-0 \mathrm{~cm}^{-1}$ with $E_{i}=1210$ $\mathrm{cm}^{-1}$. Reprinted from [38] with permission of the PCCP Owner Societies.

The ability to access small values of momentum transfer has an additional benefit. As explained earlier, INS spectra are generally recorded at low temperature, $<30 \mathrm{~K}$, to minimise the Debye-Waller factor. For many systems, this is a disadvantage because it precludes studies of reaction kinetics. From Equation (1), the Debye-Waller factor depends on both the amplitude of motion and $Q$. Thus by only using small $Q$ data, the effect of the Debye-Waller factor can be reduced. This procedure was used to study the oxidation of $\mathrm{CO}$ to $\mathrm{CO}_{2}$ over a model catalyst [40]. The time resolution was very modest, tens of minutes, but with further advances in instrumentation, operando measurements will become a regular feature of INS spectroscopy. 


\section{Conclusions}

We strongly believe, that with instrumentation such as VISION, INS spectroscopy has evolved to the stage that with sufficient sample $(1-10 \mathrm{mmol})$ and sufficient patience $(24$ hours at most), INS can measure the solid state spectrum of any material.

Even with new sources now operating, neutrons are a scarce resource. To help address this, the major institutions operate an 'open data' system, where after an embargo period, the data is made available to anyone. There is also a database of compounds that have been run at ISIS on TOSCA and its predecessor, TFXA. This can be found at: http://wwwisis2.isis.rl.ac.uk/INSdatabase/.

Although only alluded to here, the other key development has been the ability to analyse ever more complex data. This has been enabled by both increasing computer power and the availability of computational codes whose use is not restricted to dedicated experts. The combination of advanced computational methods and the recent developments we describe in this article have resulted in a 'golden age' for INS spectroscopy.

\section{Acknowledgements}

The STFC Rutherford Appleton Laboratory is thanked for access to neutron beam facilities. Computing resources (time on the SCARF compute cluster for the CASTEP calculations) was provided by STFC's e-Science facility. The INS studies, in part, used resources at the Spallation Neutron Source, a DOE Office of Science User Facility operated by the Oak Ridge National Laboratory.

\section{References}

1) P.C.H. Mitchell, S.F. Parker, A.J. Ramirez-Cuesta and J. Tomkinson, Vibrational Spectroscopy with Neutrons, with Applications in Chemistry, Biology, Materials Science and Catalysis, World Scientific, Singapore, 2005.

2) G. J. Kearley and M. R. Johnson. Vib. Spec. 53 (2010) 54-59.

3) K. Refson and S. F. Parker, ChemistryOpen 4 (2015) 520-625.

4) http://neutronsources.org/neutron-centres.html

5) A. P. J. Stampfl, A. Eltobaji, F. Darmann and K. C. Rule, Neutron News 27 (2016) 27-29.

6) $\quad$ https://www.ill.eu/

7) http://www.isis.stfc.ac.uk/

8) https://neutrons.ornl.gov/

9) $\quad$ http://j-parc.jp/researcher/index-e.html

10) https://europeanspallationsource.se/

11) A. Fedrigo, D. Colognesi, M. Bertelsen, M. Hartl, K. Lefmann, P. P. Deen, M. Strobl, F. Grazzi and M. Zoppi, Rev. Sci. Instrum. 87 (2016) 065101.

12) A. Ivanov, M. Jiménez-Ruiz and J. Kulda, J. Phys.: Conf. Series 554 (2014) 012001.

13) S. F. Parker, F. Fernandez-Alonso, A. J. Ramirez-Cuesta, J. Tomkinson, S. Rudic, R. S. Pinna, G. Gorini and J. Fernández Castañon, J. Phys.: Conf. Series, 554 (2014) 012003. 
14) https://neutrons.ornl.gov/sites/default/files/Instrument_16b_0.pdf

15) S. F. Parker, J. L. Parker and M. Jura, J. Phys. Chem. C 121 (2017) 12636-12642.

16) S. F. Parker, K. P. J. Williams, M. Bortz and K. Yvon, Inorg. Chem. 36 (1997) 52185221.

17) S. F. Parker and J. B. Forsyth, J. Chem. Soc. Faraday Trans. 94 (1998) 1111-1114.

18) T. J. Bandosz, M. Seredych, E. Rodriguez-Castellon, Y. Q. Cheng, L. L. Daemen and A. J. Ramirez-Cuesta, Carbon 96 (2016) 856-863.

19) S. F. Parker and P. M. Collier, Johnson Matthey Technical Review 60 (2016) 132144.

20) P. W. Albers. D. Lennon and S. F. Parker in "Neutron Scattering - Applications in Biology, Chemistry, and Materials Science", F. Fernandez-Alonso and D.L. Price (eds.), Academic Press, 2017, Vol 49, Ch. 5, 279-348.

21) S. F. Parker, A. J. Ramirez-Cuesta, P. W. Albers and D. Lennon, J. Phys.: Conf. Series, 554 (2014) 012004.

22) S. F. Parker, D. Lennon and P. W. Albers, Applied Spectroscopy 65 (2011) 13251341.

23) D. Lennon and S. F. Parker, Accounts of Chemical Research 47 (2014) 1220-1227.

24) A. R. McFarlane, I. P. Silverwood, R. Warringham, E. L. Norris, R. M. Ormerod, C. D. Frost, S. F. Parker and D. Lennon, RSC Advances 3 (2013) 16577-16589 and references theirin.

25) X. Liao, R. Gerdts, S. F. Parker, L. Chi, Y. Zhao, M. Hill, J. Guo, M. O. Jones and Z. Jiang, Phys. Chem. Chem. Phys. 18 (2016) 17311-17319.

26) R. Warringham, A. R. McFarlane, D. A. MacLaren, P. B. Webb, R. P. Tooze, J. Taylor, R. A. Ewings, S. F. Parker and D. Lennon, J. Chem. Phys. 143 (2015) 174703 16589 and references theirin.

27) A. R McFarlane, J. M. McNamara, H. Geller, I. P. Silverwood, R. I. Cooper, D. J. Watkin, C. D. Frost, S. F. Parker, J. M. Winfield and D. Lennon, Phys. Chem. Chem. Phys. 18 (2016) 17210-17216

28) A. J. O'Malley, S. F. Parker, A. Chutia, I. P. Silverwood, V. García Sakai and C. R. A. Catlow, Chem. Comm. 52 (2016) 2897-2900.

29) R. F. Howe, J. McGregor, S. F. Parker, P. Collier and D. Lennon, Catalysis Letters 46 (2016) 1242-1248.

30) Suwardiyanto, R. F. Howe, E. K. Gibson, C. R. A. Catlow, A. Hameed, J. McGregor, P. Collier, S. F. Parker and D. Lennon, Faraday Discussions 197 (2017) 447 - 471.

31) A. J. Elliott, B. Sakakini, J. Tabatabaei, K. C. Waugh, F. W. Zemicael and R. A. Hadden, J. Chem. Soc. Faraday Trans. 91 (1995) 3659-3662.

32) A. Würtz, Comptes Rendus Hebd. Seances Acad. Sci. 18 (1844) 702-704.

33) P. A. Korzhavyi, I. L. Soroka, E. I. Isaev, C. Lilja and B. Johansson, Proc. Natl. Acad. Sci. U.S.A. 109 (2012) 686-689.

34) E. L. Bennett, T. Wilson, P. J. Murphy, K. Refson, A. C. Hannon, S. Imberti, S. K. Callear, G. A. Chass and S. F. Parker, Inorg. Chem. 54 (2015) 2213-2220.

35) H. Auer and H. Kohlmann, Z. Anorg. Allg. Chem. 640 (2014). 3159-3165.

36) E. L. Bennett, T. Wilson, P. J. Murphy, K. Refson, A. C. Hannon, S. Imberti, S. K. Callear, G. A. Chass and S. F. Parker, Acta Cryst. B 71 (2015) 608-612. 
37) S. F. Parker, S. M. Bennington, A. J. Ramirez-Cuesta, G. Auffermann, W. Bronger, H. Herman, K. P. J. Williams and T. Smith. J. Am. Chem. Soc. 125 (2003) 1165611661.

38) T. Kandemir, M. Friedrich, S. F. Parker, F. Studt, D. Lennon, R. Schlögl and M. Behrens, Phys. Chem. Chem. Phys. 18 (2016) 17253-17258.

39) A. Chutia, I. P. Silverwood, M. R. Farrow, D. O. Scanlon, P. P. Wells, M. Bowker, S. F. Parker, C. R. A. Catlow, Surf. Sci. 653 (2016) 45-54.

40) S. F. Parker, Chem. Comm. 47 (2011) 1988-1990. 